$\mathrm{Hg}^{1}$. For transcriptome analysis, total RNAs from whole peripheral blood cells were extracted with using PAXgene miRNA kit. After constructing single-stranded, strand-specific libraries, multiplex sequencing was done. After quantifying the expressions of transcripts, differentially expressed genes (DEGs) between exPH and exN group were selected by paired T-test $(P<0.05)$. And then, hierarchical clustering analysis and pathway enrichment analysis (PathVisio) were performed. Results: There were no significant differences between exPH and exN group in the result of total skin score, serum BNP, tests of pulmonary function and thermography after $0^{\circ} \mathrm{C}$-stress. Positive SSc-related autoantibody was a risk factor for exPH (odds ratio, 1.41); especially, positive anti-RNP seemed to be prominent (odds ratio, 3.21). Based on the 817 DEGs between exPH and exN group, the hierarchical clustering showed major 4 clusters, and one of them consisted of only cases in exPH group. When we focused on 117 genes reported to be directly implicated in the development of $\mathrm{PAH}^{2}$ ), it is noteworthy that 4 of them including TGF- $\beta$ induced protein were differentially expressed. Pathway analysis of transcriptome revealed that 22 pathways, such as hypertrophy model, lung fibrosis and Wnt/B-catenin signaling, were differently enriched between exPH and exN group.

Conclusions: The paradigm of SSc-PAH management should ideally be aimed at detecting early PVD and starting treatment prior to fulfilling the criteria for PAH. Although detection of early PVD in SSc patients remains a major challenge, exercise DE seemed to be a good, non-invasive method for screening. It is noteworthy that expression changes in some of known PAH-related genes were detected from peripheral blood of exPH patients. It shows the possibility that the therapeutic intervention at early stage of the disease may alter the clinical course. References:

[1] R. Naeije et al., Am. J Resp. \& Critical Care Med. 187, 576-583 (2013). .

[2] Parikh VN et al., Circulation 125, 1520-1532 (2012).

Disclosure of Interest: None declared

DOI: 10.1136/annrheumdis-2017-eular.3627

\section{FRI0418 FACTORS ASSOCIATED WITH STEROID-FREE REMISSION IN PATIENTS WITH INFLAMMATORY MYOPATHIES. A RETROSPECTIVE ANALYSIS OF A SINGLE-CENTER COHORT}

Y. Sato ${ }^{1}$, K. Kobayashi ${ }^{1}$, K. Minegishi ${ }^{1}$, S. Ohno ${ }^{1}$, H. Nakajima ${ }^{2}{ }^{1}{ }^{1}$ Center for Rheumatic Diseases, Yokohama City University Medical Center; ${ }^{2}$ Department of Stem Cell and Immune Regulation, Yokohama City University Graduate School of Medicine, Yokohama, Japan

Background: The inflammatory myopathies are a heterogeneous group of connective tissue diseases characterized by muscle weakness and inflammation. Corticosteroids are the standard main treatment for inflammatory myopathies. However, steroid therapy often causes a wide range of side effects. Although immunosuppressive drugs are used as steroid-sparing agents in an effort to prevent disease recurrence, the appropriate duration of steroid use remains unclear.

Objectives: We investigated whether steroid therapy can be safely withdrawn in patients with inflammatory myopathies followed in a single center.

Methods: We retrospectively reviewed clinical charts of 71 consecutive patients (age 51.9 \pm 15.7 y.o., female 69\%) who met Bohan and Peter criteria for polymyositis (PM)/dermatomyositis (DM) and modified Sontheimer's criteria for clinically amyopathic dermatomyositis (ADM), respectively. Steroid free remission was defined as a 3-month consecutive period of no disease activity without corticosteroid treatment. Factors associated with steroid free remission were examined.

Results: Of 71 identified patients, 29 patients $(40 \%)$ were DM, 15 patients $(21 \%)$ were PM, 9 patients $(13 \%)$ were overlap myositis, and 18 patients $(25 \%)$ were ADM. Thirty-seven patients $(52 \%)$ had muscle weakness, 5 patients $(7 \%)$ had malignancies and 43 patients $(61 \%$ ) had signs of interstitial lung disease. With a mean follow-up of $6.6 \pm 5.0$ years, 9 patients $(13 \%)$ died during follow-up period. The remaining 62 patients were treated with corticosteroids alone or in combination with immunosuppressants. Steroid-free remission was achieved in 21 of 62 patients $(34 \%)$ patients with a mean time to steroid withdrawal of $5.5 \pm 4.0$ years. Six of 21 patients $(29 \%)$ relapsed $1.7 \pm 1.7$ years after steroid withdrawal. There were no differences in onset of age, disease duration, positive ANA, positive Anti Jo-1 antibodies, serum creatine kinase levels, maximum dose of corticosteroids, skin, joint and lung involvement between steroid-free group and non-steroid-free group. Elevated inflammatiory markers were associated with long-term steroid use $(p=0.038)$. Concominant immunosuppressants were more frequently used in non-steroid-free group than steroid-free group $(p=0.002)$.

Conclusions: Steroid-free remission might be achieved in some patients with inflammatory myopathies.

Disclosure of Interest: None declared

DOI: 10.1136/annrheumdis-2017-eular.4750

\section{FRI0419 THE PREDICTIVE PROGNOSTIC FACTORS FOR CLINICAL COURSE OF POLYMYOSITIS/DERMATOMYOSITIS- ASSOCIATED INTERSTITIAL LUNG DISEASE}

Y. Sugiyama ${ }^{1}$, R. Yoshimi ${ }^{1}$, M. Tamura ${ }^{1}$, N. Hamada ${ }^{1}$, H. Nagai ${ }^{1}$, N. Tsuchida ${ }^{1}$, Y. Soejima $^{1}$, Y. Kunishita ${ }^{1}$, D. Kishimoto ${ }^{1}$, H. Nakano ${ }^{1}$, R. Kamiyama ${ }^{1}$, K. Minegishi ${ }^{2}$, Y. Asami ${ }^{1}$, Y. Kirino ${ }^{1}$, S. Ohno ${ }^{2}$, H. Nakajima ${ }^{1}$ on behalf of Y-CURD Study Group. ${ }^{1}$ Department of Stem Cell and Immune Regulation, Yokohama City University Graduate School of Medocine; ${ }^{2}$ Center for Rheumatic Diseases, Yokohama City University Medical Center, Yokohama, Japan

Background: Interstitial lung disease (ILD) and concomitant infectious diseases are the predominant causes of death in polymyositis/dermatomyositis (PM/DM). We have already reported that hypocapnea and ILD lesion in upper lung fields are independent prognostic factors. Micro RNA is a non-coding RNA, which has a certain function such as transcriptional regulation. miR-1 has been reported to be associated with myocyte differentiation and to decrease in muscle tissue from patients with inflammatory myopathies.

Objectives: Here we investigated the association of serum miR-1 level with clinical course of PM/DM-associated ILD (PM/DM-ILD).

Methods: We retrospectively analyzed clinical baseline, serum miR-1 level, initial therapeutic regimens, total amounts of PSL, clinical outcomes, and episode of infection of patient with PM/DM-ILD who had received initial treatment at six hospitals associated with Yokohama City University from 2003 to 2016. The serum miR-1 level was measured by quantitative real-time PCR.

Results: One hundred sixteen (PM 22, DM 51, and clinically amyopathic DM 43) patients were included. The mean age was $56 \pm 15$ years and 83 were female. As initial therapies, oral PSL, methylprednisolone (mPSL) pulse, intravenous cyclophosphamide (IVCY), and oral calcineurin inhibitor therapies were performed in $113(97 \%), 80(69 \%), 48(41 \%)$ and $80(69 \%)$, respectively. Forty-one patients had a serious infection at $51 \pm 38$ days from initiation of immunosuppressants and 10 died of infections. Old age, low $\mathrm{PaCO}_{2}$ and albumin, high LDH and KL-6, high score of ILD, high initial dose of PSL, mPSL pulse, IVCY, calcineurin inhibitor and combination therapy were extracted as risk factors for infection by univariate analyses. A multivariate logistic regression analyses revealed that combination therapy $(p=0.012$, OR 2.83$)$, old age $(p=0.024$, OR 2.12), high initial dose of PSL ( $p=0.024$, OR 2.69), low albumin ( $p=0.031$, OR 3.56), and low $\mathrm{PaCO}_{2}(p=0.038$, OR 2.67) were independent risk factors for infection. Serum samples were obtained from total of 14 patients and 13 healthy controls. Serum miR-1 levels in PM/DM-ILD patients before treatment were significantly higher than those in healthy controls $(p=0.047)$. Also serum miR-1 levels were significantly higher in PM/DM-ILD patients with concomitant infectious diseases as compared to patients without infectious diseases $(p=0.043)$. We further divided the PM/DM-ILD cases into two groups by the serum miR-1 level. The higher miR-1 group showed poorer effectiveness of ILD treatment $(p=0.040)$, and lower lymphocyte count $(p=0.013)$ as compared to the lower miR-1 group.

Conclusions: Appropriate monitoring is important for PM/DM-ILD, especially in older patients with malnutrition or decreased respiratory function. miR-1 can be a new biomarker for predicting treatment response and concomitant infectious diseases during treatment for PM/DM-ILD.

References:

[1] Robert W. Georgantas et al, Inhibition of myogenic microRNAs 1, 133, and 206 by inflammatory cytokines links inflammation and muscle degeneration in adult inflammatory myopathies, Arthritis Rheum, 2014;66:1022-33.

Disclosure of Interest: None declared

DOI: 10.1136/annrheumdis-2017-eular.3168

\section{FRIDAY, 16 JUNE 2017}

\section{Spondyloarthritis - etiology, pathogenesis and animal models}

\section{FRI0420 ASSOCIATION OF SUPRESSOR OF CYTOKINE SIGNALING -3 (SOCS-3) EXPRESSION WITH INTERLEUKIN-23 RECEPTOR (IL-23R) SINGLE NUCLEOTIDE POLYMORPHISMS (SNPS) IN ANKYLOSING SPONDYLITIS (AS)}

M.A. Sánchez ${ }^{1}$, R. Villares ${ }^{2}$, J. Polo y La Borda ${ }^{3}$, J. Campos ${ }^{3}$, J.M. Rodríguez-Frade ${ }^{2}$, J. Sanz ${ }^{3}$, B.J. Robles Flores ${ }^{3}$, A. Royuela ${ }^{4}$, P. Lucas $^{2}$, M. Mellado ${ }^{5}$, J. Mulero ${ }^{3} \cdot{ }^{1}$ Rheumatology, Instituto de Investigación Biomédica Hospital Universitario Puerta de Hierro Majadahonda, Majadahonda (Madrid); ${ }^{2}$ Immunology and Oncology Department, Centro Nacional de Biotecnología. CNIC, Madrid; ${ }^{3}$ Rheumatology; ${ }^{4}$ Clinical Biostatistics Unit, Instituto de Investigación Biomédica Hospital Universitario Puerta de Hierro Majadahonda; ${ }^{5}$ Immunology and Oncology Department, Centro Nacional de Biotecnología. CNIC, Majadahonda (Madrid), Spain

Background: Nowadays genetic-association studies have discovered new genes, other than $H L A-B 27$, as $I L-23 R$ associated with AS. The signalling pathway trough IL-23R is negatively regulated by the SOCS proteins. However, the reports regarding the roles of SOCS in AS are very rare at present..$^{1,2}$

Objectives: The aim of this study is to assess the gene expression of SOCS-1, 